\title{
Transdermal delivery of combined hormonal contraception: a review of the current literature
}

\author{
This article was published in the following Dove Press journal: \\ International Journal of Women's Health \\ 15 May 2017 \\ Number of times this article has been viewed
}

\author{
Rosanna M Galzote' \\ Sally Rafie ${ }^{2}$ \\ Rachel Teal' \\ Sheila K Mody' \\ 'Section of Family Planning, \\ Department of Reproductive \\ Medicine, University of California, \\ San Diego, ${ }^{2}$ Department of Pharmacy, \\ UC San Diego Health, San Diego, \\ CA, USA
}

\begin{abstract}
The transdermal patch provides an effective and convenient option for hormonal contraception. The patch currently on the US market contains $150 \mu \mathrm{g}$ norelgestromin and $35 \mu \mathrm{g}$ ethinylestradiol (EE). The $20 \mathrm{~cm}^{2}$ patch is applied once weekly for 3 weeks, followed by a patchfree week, for a 21-7 cycle. Typical failure rates are similar to that of combined oral contraceptives (COCs). Transdermal delivery results in less peaks and troughs of estrogen, but a higher total estrogen exposure compared with COCs. Though studies show mixed results, the risk of developing venous thromboembolism (VTE) is about twice as high with the patch as with COCs; however, the absolute risk of VTE remains low. The side effect profile is similar to that of COCs, with slightly higher rates of breast tenderness plus a unique adverse effect of application site reactions. Two new patches have been developed, one containing gestodene and EE in Europe and another containing levonorgestrel and EE. Overall, the patch provides an alternative to COCs for women who want autonomy and the benefit of not needing to take a pill daily, with similar efficacy and tolerability. Keywords: contraceptive patch, Ortho-Evra, transdermal, levonorgestrel patch, gestodene patch, hormonal patch
\end{abstract}

\section{Background}

Since the development of the oral contraceptive (OC) pill in the 1960s, hormonal contraception has taken many forms. Combined hormonal contraception (CHC), referring to methods with both estrogen and progestin, can be delivered orally, transdermally, or transvaginally. Although long-acting reversible contraception (LARC) has become more popular, there is still a desire from patients to have a contraceptive method they can control. Additionally, hormonal contraception offers benefits not seen with some of the LARC methods, including improved cycle control and acne treatment. The transdermal and transvaginal contraceptive options give patient autonomy and the benefit of not needing to use the method daily.

The first transdermal delivery system developed in the 1980 s was a scopolamine patch. Since then, medications that have been developed in a transdermal form include nicotine, estradiol for hormone therapy, fentanyl, clonidine, nitroglycerin, among others. For successful delivery of a medication through a transdermal system, the molecule must be small and lipophilic to permeate through the skin. Estradiol and ethinylestradiol (EE) are ideal molecules as therapeutic levels can be delivered easily, whereas progesterone and progestins require higher therapeutic levels. ${ }^{1}$ The first transdermal contraceptive patch on the US market, Ortho Evra ${ }^{\mathrm{TM}}$ (Ortho-McNeilJanssen Pharmaceuticals Inc., Titusville, NJ, USA), was approved by the US Food and Drug Administration in November 2001.

A transdermal patch offers a number of benefits compared with OCs. There is less variability in plasma concentrations of estrogen, which may decrease estrogen-related 
side effects that result from high peak estrogen levels, such as nausea. Though peaks and troughs are minimized, overall estrogen exposure as measured by the area under the concentration curve (AUC) is higher with the Ortho Evra patch compared with the combined oral contraceptives (COCs). A second major advantage is that the user only changes the patch once weekly, as opposed to taking a contraceptive pill daily, which could result in improved adherence. A pooled study of 1,785 patch users showed perfect use ranging from $88.1 \%$ to $91.0 \%$ across different age groups. In this study, age did not affect adherence. Another study has reported larger differences across age groups. ${ }^{2}$ In contrast, perfect use of COCs ranged from $67.7 \%$ to $85.2 \%$ and differed significantly by age, with lowest rates in $<20$-year-old females. ${ }^{3}$

\section{Pharmacology and pharmacodynamics}

The Ortho Evra contraceptive patch is a $20 \mathrm{~cm}^{2}$ adhesive that releases $35 \mu \mathrm{g}$ EE and $150 \mu \mathrm{g}$ norelgestromin (NGMN) per day. ${ }^{4} \mathrm{NGMN}$ is an active metabolite of norgestimate, the progestin contained in the OCs Ortho-Cyclen ${ }^{\circledR}$ and Ortho Tri-Cyclen ${ }^{\circledR}$. ${ }^{4}$ During development of this product, three patch sizes, 10,15 , and $20 \mathrm{~cm}^{2}$, were compared in a study of 610 subjects. It was found that the $20 \mathrm{~cm}^{2}$ patch achieved ovulation suppression and cycle control similar to that of Ortho-Cyclen (6.2\% $20 \mathrm{~cm}^{2}$ patch, 7.2\% Ortho-Cyclen); thus, the only size patch available is the $20 \mathrm{~cm}^{2} .^{5}$

Reference ranges were set at $0.6-1.2 \mathrm{ng} / \mathrm{mL}$ for NGMN and 25-75 pg/mL for EE, as a developmental tool to assess efficacy. The concentrations at steady state are $0.83 \mathrm{ng} / \mathrm{mL} \pm 0.21$ for $\mathrm{NGMN}$ and $56.7 \mathrm{pg} / \mathrm{mL}$ for $\mathrm{EE}$, both of which are within the set reference ranges. ${ }^{4}$ Compared to the peaks and troughs seen in serum concentrations with the pill, the patch maintains a steadier concentration throughout the day. Serum levels of each stayed within the reference range for the entirety of the 7-day period in the patch's first cycle. Serum levels of NGMN and EE were 20\% less if worn on the abdomen compared with the buttock, thigh, or upper arm, though at all sites, the concentration remained within the reference ranges. The mean serum levels of NGMN and EE also remained within the reference range in conditions of heat, humidity, exercise, and cool-water immersion. ${ }^{4}$

One study done in the Netherlands compared mean serum EE concentrations in subjects using the patch $(20 \mu \mathrm{g} E \mathrm{E} /$ day $)$, COC (30 $\mu \mathrm{g}$ EE/day), and NuvaRing ${ }^{\circledR}$ (Merck \& Co., Kenilworth, NJ, USA; $15 \mu \mathrm{g}$ EE/day). Concentration over time was more variable in COCs compared with the patch and NuvaRing, as the pill had higher peak concentration $\left(\mathrm{C}_{\max }\right)$ of 4.5 times than that of the patch and 1.6 times than that of the NuvaRing. The overall exposure to EE, measured by the mean $\mathrm{AUC}_{0-21}$, was highest for the patch that was 3.4 times that of NuvaRing and 1.6 times that of COCs. ${ }^{6}$

The mechanism of action of NGMN and EE involves 1) thickening the cervical mucus to prevent sperm penetration, 2) decreasing the endometrial receptivity to reduce likelihood of implantation, and 3 ) inhibiting ovulation by suppressing gonadotropins, follicle-stimulating hormone (FSH), and luteinizing hormone (LH). ${ }^{7}$ Steady state concentration is reached within 2 weeks of patch use, though pregnancy prevention is achieved after 1 week. The half-lives of NGMN and EE are 28.4 and 15.2 hours, respectively. Mean FSH, $\mathrm{LH}$, and estradiol values return to baseline levels 6 weeks after discontinuation. ${ }^{7}$

\section{Efficacy}

An initial open-label 73-center study in 2001 reported an overall failure rate of $0.7 \%$ and a method-failure rate of $0.4 \%$ through 13 cycles for transdermal delivery. The Pearl index (PI), or number of pregnancies per 100 woman-years, was 0.71 for overall failure and 0.59 for method failure. ${ }^{8}$ Similar numbers were reflected in one subsequent study of pooled data from three studies in 3,319 women. Failure rates were $0.8 \%$ overall and $0.6 \%$ from method failure, corresponding to PIs of 0.88 and 0.7 , respectively. ${ }^{9}$

In a large epidemiological trial in the UK, patients prescribed Evra ${ }^{\mathrm{TM}}$ had an incidence of 0.34 unintended pregnancies per 100 women-years. This was higher than the rate with second-generation COCs of 0.16 and 0.12 for third-generation COCs, but lower than progestin-only pills at 0.43 . This case-control study was limited as it analyzed prescriptions of contraception, though did not assess actual use of each method. ${ }^{10}$ Pooled data of 812 Ortho Evra patch users in the US in a 2004 study showed the impact of compliance on contraceptive efficacy. With perfect compliance, the PI was 0.73 . Imperfect dosing increased failure rates to a PI of 2.33 in patch users. They also found that overall, there was a significantly higher proportion of cycles with perfect dosing in patch use compared to OC use (88.7\% vs $79.2 \%){ }^{11}$

\section{Body weight}

There was concern over decreased efficacy of transdermal patches in women with higher body weights. Pooled data from three multicenter studies showed significantly increased rates of unintended pregnancy in women $\geq 90 \mathrm{~kg}$. In women $<90 \mathrm{~kg}$, there was no significant association between body weight and pregnancy. Hormone levels decreased with higher body 
weight, but analyses showed that only $10 \%-20 \%$ of variability was attributed to body weight. ${ }^{9}$

A prospective study of contraceptive failures in 1,523 CHC users with a high sample size of obese and overweight females did not show body mass index (BMI) to be a significant risk factor for unintended pregnancy. Three-year failure rates did not differ across different BMI categories among CHC users (BMI <25: 8.44\%, BMI 25-30: 11.05\%, and $\mathrm{BMI}>30: 8.92 \%$ ). Failure rates were similar across the three methods: COC, 5.6\%; patch, 4.6\%; and vaginal ring, 3.4\% $(P=0.22)$. It is postulated that reduced fertility with increased BMI explains the similar rates of contraception failure. ${ }^{12}$

\section{Safety}

As with any contraceptive containing estrogen, there is a slightly increased risk of developing venous thromboembolism (VTE) with the patch relative to women not on hormonal contraceptives. Given the overall higher exposure to estrogen with the patch $(60 \%$ greater AUC) compared to COCs, there was concern that this could translate to an increased risk of thromboembolism events compared to women using pills. ${ }^{13}$

In a postmarketing case-control study published in 2006 by Jick et al, ${ }^{13}$ nonfatal VTE risk was compared in Ortho Evra patch users and users of the norgestimate-35 (NGM-35) OC, containing norgestimate and $35 \mu \mathrm{g}$ EE, between 2002 and 2004. They found an overall incidence rate for VTE of 52.8 per 100,000 women-years in patch users and 41.8 per 100,000 women-years in NGM-35 OC users. The odds ratio (OR) for VTE was 0.9 for contraceptive patch users compared to NGM-35 users. ${ }^{13}$ A follow-up postmarketing study that included cases up to 2007 found a higher OR of 2.0 (95\% CI 0.9-4.1) between patch and COC users, but concluded that the patch does not confer statistically significant excess risk of VTE compared to NGM-35 COC users. ${ }^{14}$

Findings of a study by Cole et $\mathrm{al}^{15}$ drew different conclusions. The case-control study using private insurance claims data found a significantly increased risk of VTE, myocardial infarction, or ischemic stroke in patch users compared to users of norgestimate-containing COC with $35 \mu \mathrm{g}$ EE from 2002 to 2004 . There was an incidence ratio of 2.2 (95\% CI 1.3-1.8) for VTE, with incidences of 40.8 cases per 100,000 woman-years in patch users, compared to 18.3 per 100,000 in norgestimate-containing COC users. ${ }^{15}$ A study update by Dore et $\mathrm{al}^{16}$ had consistent findings. They again found an OR of 2.0 for VTE compared with users of NGM-35 that was significant. ${ }^{16}$ The ORs were $0.6(95 \%$ CI $0.1-3.2)$ for stroke and 1.2 (95\% CI 0.3-4.7) for acute myocardial infarction
(AMI). The incidence of stroke and AMI was low, making it difficult to understand the precise risk. ${ }^{16}$ Due to these concerns over increased risk of thrombotic events, a black box warning was released by the US Food and Drug Administration (FDA) in 2004 for Ortho Evra labeling and updated again most recently in 2011.

\section{Utilization}

In 2013, 1.6\% of women aged 15-44 years in the US used the ring or patch, and $2.6 \%$ of women using contraception used either the ring or patch. ${ }^{17}$ In a study of focus groups of young women, negative attitudes toward the patch include distrust of effectiveness, as they are not familiar with this method of drug delivery, whereas a pill is more widely accepted as a reliable route. They feared the patch may fall off and held concerns about visibility. There was also concern regarding safety and the increased risk of blood clots in this population. However, many of the women did agree that the patch was easier to remember to use compared to pills. ${ }^{18}$

\section{Tolerability}

Adverse effects of the patch are similar to those of COCs. The most commonly reported complaints and reasons for discontinuation are mild-to-moderate in severity and include application site reactions, nausea, emotional lability, headache, and breast discomfort. ${ }^{8,19}$ Weight gain is minimal in patch users, similar to COC users $-87.8 \%$ of patch users stayed within $5 \%$ of baseline body weight. ${ }^{19}$ Rates of unscheduled, or breakthrough bleeding (BTB) and spotting were low $(<10 \%$ BTB, $<20 \%$ BTB $/$ spotting) and decreased with continued use. ${ }^{9}$ Application site reaction is one adverse event unique to the patch. In a pooled study of 812 patch users, there was a $17.4 \%$ overall incidence of application site reaction, causing discontinuation in $1.9 \%$. Most reactions (91.4\%) were mild-to-moderate in severity. ${ }^{19}$ Breast symptoms include breast discomfort, engorgement, and pain. About one-fifth of patch users experienced breast symptoms, mostly in the first two cycles. They were mild-tomoderate in severity in most and decreased over time. Breast symptoms were treatment limiting in $1.9 \%$ of participants in a pooled study. In a comparative study, breast symptoms were three times more prevalent in patch users than COC users ( $18.8 \%$ vs $1.6 \%)$, but declined to similar rates after the second cycle. ${ }^{19}$ Another concern patients express unique to the patch is detachment. The detachment rate is $4.7 \%$ with $1.8 \%$ being fully detached and $2.9 \%$ partially detached. A study shows that adhesion does not differ in conditions of increased heat and humidity or with exercise. Patients are advised to replace patches if they become fully or partially detached. ${ }^{20}$ 


\section{Patient satisfaction}

In a study of continuation and satisfaction at 12 months of women aged 14-45 years in the Contraceptive CHOICE Project, continuation rates were lowest for the patch at $49.1 \%$ compared to other contraceptive methods $(55.1 \%$ for COCs to $87.5 \%$ for LNG-IUD). Only $35.1 \%$ of women using the patch reported being very satisfied with the method and $55.7 \%$ were not satisfied..$^{21}$ The most common reasons for discontinuation of the patch in women in the CHOICE study were not liking "side effects" and logistical reasons. Approximately $41 \%$ of patch discontinuers reported side effects. ${ }^{21}$

In contrast, in a small study of 28 adolescents who started Ortho Evra in 2002-2003, 68\% were very satisfied and $29 \%$ were somewhat satisfied, with $93 \%$ stating they would recommend the method to a friend/relative. Despite high satisfaction rates, adolescents discontinue the patch at higher rates than COCs. ${ }^{22}$ A prospective longitudinal study comparing adolescent use of contraceptive patch versus pills in 2011 showed after nine cycles that $38 \%$ of patch users compared to $60 \%$ of pill users had continued the method initiated at enrollment. This is despite both methods having similar satisfaction and patch users reporting that their method improved normal daily activities. ${ }^{23}$

The 1-year contraceptive continuation has been shown to be low among adolescents. In a 12-month longitudinal cohort study of 1,387 women aged 15-24 years, the patch had the lowest continuation rate at follow-up of only $10.9 \%$ compared to $32.7 \%$ in pill initiators. Additionally, the pregnancy rate in this study was second highest for patch with a PI (pregnancies per 100 person-years) of 30.1. ${ }^{24}$ This low rate of continuation in young women was consistent with another study in 2015 of 130 adolescents aged 13-20 years. When offered intrauterine devices (IUDs), injectable, COC, patch, and the ring, $13 \%$ opted for the patch. Six-month continuation rates were lowest with the patch and ring at $17 \%$, compared to $88 \%$ with the IUD, $20 \%$ with the injectable, and $43 \%$ with COCs. Of the 23 who chose the patch or ring, 11 never initiated, 2 continued, and 10 discontinued. ${ }^{25}$ It is important to note that this was a small study and reasons for not initiating or discontinuing methods were not reported.

\section{Current patch}

Many of the aforementioned studies are on the Ortho Evra patch which has been on the market in the US, but the Evra patch, used in European countries and Canada, has also been studied extensively. ${ }^{26}$ This $20 \mathrm{~cm}^{2}$ adhesive contains $600 \mu \mathrm{g}$ EE and $6 \mathrm{mg}$ NGMN, releasing 33.9 $\mu \mathrm{g}$ EE and $203 \mu \mathrm{g}$ NGMN per day. Studies show that Evra users have higher satisfaction and compliance rates than $\mathrm{COC}$ users. ${ }^{27,28}$ Although the relative risk for any VTE has shown to be 2.0 compared to corresponding $\mathrm{COCs},{ }^{29}$ the Evra patch did not have the same widespread VTE scare as the Ortho Evra patch did in the US.

\section{New patches EE/GSD}

A novel transparent patch has been developed by Bayer that delivers $0.5 \mathrm{mg}$ EE and $2.1 \mathrm{mg}$ gestodene (GSD). GSD is a progestin contained in many COCs widely used in Europe for years. It is a favorable drug for transdermal use as it is has an established efficacy and safety profile, and good skin absorption allowing for a low dose needed and small patch size. The dosing of this $11 \mathrm{~cm}^{2}$ patch results in the same amount of hormone exposure as the $0.02 \mathrm{mg} \mathrm{EE}$ and $0.06 \mathrm{mg}$ GSD OC. This dosage was justified in a Phase IIa study that showed ovulation inhibition is not as effective with half the dose of estrogen or progestin. ${ }^{30}$ The EE/GSD patch has decreased EE exposure measured by the AUC compared to the EE/NGMN patch. ${ }^{31}$ Similar to the EE/NGMN patch, there is a 7-day application period for 3 weeks with 1 week patch-free (21/7). In a Phase III uncontrolled, open-label study, the EE/GSD patch had an unadjusted PI of 1.19 and an adjusted PI of 0.81 for pregnancy due to noncompliance. Of those originally enrolled, $14.3 \%$ discontinued due to an adverse event. Of those who stayed in the study, compliance was high with a mean of $97.9 \%$ and a median of $100 \%$. At least one adverse event was reported in $61.7 \%$ of subjects, the most common being headache $(9.5 \%)$, application site reaction $(8.5 \%)$, nasopharyngitis $(7.0 \%)$, cervical dysplasia including atypical squamous cells of undetermined significance (6.2\%), and application site erythema (4.9\%). Two of 1,631 women in the study were diagnosed with pulmonary embolism, over the course of the year of the study. ${ }^{32}$

Despite the lower EE delivery in the EE/GSD patch, bleeding patterns were shown to be similar compared to the EE/NGMN patch in a descriptive study. Withdrawal bleeding was shorter in the EE/GSD patch group in the first seven cycles with similar intensity. The incidence of breast pain was slightly lower in EE/GSD users compared to tradition EE/NGMN patch users, which is expected given the total lower estrogen exposure. ${ }^{33}$ When compared to a COC containing $0.02 \mathrm{mg}$ EE and $0.1 \mathrm{mg}$ LNG in a Phase III doubleblind, double-dummy multicenter trial, bleeding cycle and patterns were comparable. ${ }^{34}$

\section{EE/LNG (AG200-I5)}

An investigational contraceptive patch, AG200-15, has been developed by Agile Therapeutics (Princeton, NJ, USA). This patch is a $15 \mathrm{~cm}^{2}$ matrix core with a surrounding adhesive 
for a total area of $26 \mathrm{~cm}^{2}$, containing $2.3 \mathrm{mg}$ EE and $2.6 \mathrm{mg}$ levonorgestrel (LNG). Major differences between this and the currently marketed NGMN/EE patch are the decreased AUC of estrogen and the use of LNG, which has been shown to have lower rates of VTE compared to other progestins. Like the other patches, each patch was applied to the skin for 7 days three times per cycle followed by one patch-free week, for a 21/7 cycle. This dosing provides similar LNG and EE serum concentrations compared to those of $20 \mu \mathrm{g}$ LNG and $30 \mu \mathrm{g}$ EE pill. ${ }^{35}$

In a Phase III open-label study including obese and nonobese women, the PI for the patch was 4.45 , compared to 4.02 for the $100 \mu \mathrm{g} \mathrm{LNG}, 20 \mu \mathrm{g}$ EE pill. Compliance was determined by a self-reporting diary and verified by LNG and EE levels. After eliminating pregnancies in women with undetectable hormone levels, the PIs were 2.82 for the patch and 3.8 for the pill, which, statistically, were not significantly different. Of note, PIs in obese and nonobese patients did not significantly differ at 4.59 and 4.40 , respectively. Self-reported compliance in this study was $91.6 \%$ in patch users and $79.8 \%$ in nonpatch users. However, the rates of laboratory-confirmed compliance for cycles 2 and 6 were $9.9 \%$ and $11 \%$ for the patch and $8.8 \%$ and $12.6 \%$ for the pill, which were not significantly different for the two methods. The discrepancy between self-reported and laboratory-verified compliance sheds light on prior studies that used patient diaries to assess compliance and may have been overestimating rates. ${ }^{36} \mathrm{~A}$ second Phase III clinical trial of the EE/LNG patch is being conducted (NCT02158572), as the first trial including obese and nonobese women had a high PI and high noncompliance rates.

Bleeding patterns were similar in the patch and pill groups with $25.4 \%$ and $23.2 \%$ of women with unscheduled bleeding or spotting, respectively. There were similar rates of discontinuation due to bleeding for each method. A total of $21.8 \%$ of patch users experienced a drug-related treatment-emergent adverse event compared with $16 \%$ in the pill group. Most treatment-emergent adverse events were mild-to-moderate in severity. The most common adverse events were estrogen related, including nausea, headache, increased weight, and breast tenderness. ${ }^{34}$ Skin reaction occurred in $3.2 \%$ of patch users, a lower rate than that seen with the traditional NGMN/EE patch. ${ }^{37}$ Low detachment rates have been reported $(2.0 \%-3.7 \%)$, with sustained wearability with exercise and in humid climates. ${ }^{38,39}$

\section{Conclusion}

Transdermal patches provide an effective and convenient method of hormonal contraception. Its once-weekly application is appealing for women who want an alternative to daily OCs. Although this offers a theoretical benefit of higher compliance and lower failure rates, efficacy is in the same range as oral and transvaginal $\mathrm{CHCs} .{ }^{40}$ Compliance rates are reported to be higher in patch users compared to pill users; although most studies use diaries or self-reporting to measure compliance, there may be some inaccuracy. Additionally, compliance and continuation are low in adolescents, so the failure rate with typical use may be higher in this population.

The side effect profile of the patch is similar to that of combined OCs, which are estrogen-related and mostly mild-tomoderate in severity. These include nausea, breast tenderness, emotional lability, and dysmenorrhea. One unique adverse effect is application site reaction, which occurs in $\sim 20 \%$ of users and is treatment-limiting in $2 \%$. Adhesion of the patch remains high in humid climates and with exercise. ${ }^{20}$

An advantage of the transdermal route is that the levels of estrogen are steady without the peaks and troughs seen with OCs, but the AUC of estrogen is higher in patch users. Given the higher total estrogen exposure, there has been concern raised over a higher risk of VTE compared to pill users. Studies are conflicting, but there is evidence that the patch confers a twofold risk of developing a nonfatal thromboembolic event compared with OCs. Although the relative risk is higher for VTE compared to OCs the absolute risk of VTE remains low. In light of this potential increased risk, two new patches with lower estrogen exposure based on AUC and different progestins are under investigation. One is a smaller, transparent EE/GSD patch being studied in Europe. The other is the EE/LNG patch in the US that has not yet received FDA approval. It will be interesting to see if there is higher uptake, acceptability and continuation with newer patches.

\section{Disclosure}

The authors report no conflicts of interest in this work.

\section{References}

1. Potts RO, Lobo RA. Transdermal drug delivery: clinical considerations for the obstetrician-gynecologist. Obstet Gynecol. 2005;105(5 pt 1): 953-961.

2. Bodner K, Bodner-Adler B, Grünberger W. Evaluation of the contraceptive efficacy, compliance, and satisfaction with the transdermal contraceptive patch system Evra: a comparison between adolescent and adult users. Arch Gynecol Obstet. 2011;283(3):525-530.

3. Archer DF, Bigrigg A, Smallwood GH, et al. Assessment of compliance with a weekly contraceptive patch (Ortho Evra/Evra) among North American women. Fertil Steril. 2002;77(2 suppl ):S27-S31.

4. Abrams LS, Skee D, Natarajan J, Wong FA. Pharmacokinetic overview of Ortho Evra/Evra. Fertil Steril. 2002;77(2 suppl 2):S3-S12.

5. Dittrich R, Parker L, Rosen JB, et al. Transdermal contraception: evaluation of three transdermal norelgestromin/ethinyl estradiol doses in a randomized, multicenter, dose-response study. Am J Obstet Gynecol. 2002 186(1):15-20. 
6. van del Heuvel MW, van Bragt AJ, Alnabawy AL, Kaptein MC. Comparison of ethinylestradiol pharmacokinetics in three hormonal contraceptive formulations: the vaginal ring, the transdermal patch and an oral contraceptive. Contraception. 2005;72(3):168-174.

7. Ortho Evra ${ }^{\circledR}$ (norelgestromin/ethinyl estradiol transdermal system) [package insert]. Titusville, NJ: Janssen Pharmaceuticals, Inc.; 2012.

8. Smallwood GH, Meador ML, Lenihan JP Jr, et al. Efficacy and safety of a transdermal contraceptive system. Obstet Gynecol. 2001;98(5 pt 1): 799-805.

9. Zieman M, Guillebaud J, Weisberg E, Shangold GA, Fisher AC, Creasy GW. Contraceptive efficacy and cycle control with the Ortho Evra/ Evra transdermal system: the analysis of pooled data. Fertil Steril. 2002; 77(2 suppl 2):S13-S18.

10. Jick SS, Habgerg KW, Kaye JA, Jick H. The risk of unintended pregnancies in users of the contraceptive patch compared to users of oral contraceptives in the UK General Practice Research Database. Contraception. 2008;80(2):142-151.

11. Archer DF, Cullins V, Creasy GW, Fisher AC. The impact of improved compliance with a weekly contraceptive transdermal system (Ortho Evra) on contraceptive efficacy. Contraception. 2004;69(3):189-195.

12. McNicholas C, Zhao Q, Secura G, Allsworth JE, Madden T, Peipert JF. Contraceptive failures in overweight and obese combined hormonal contraceptive users. Obstet Gynecol. 2013;121(3):585-592.

13. Jick SS, Kaye JA, Russman S, Jick H. Risk of nonfatal venous thromboembolism in women using a contraceptive transdermal patch and oral contraceptives containing norgestimate and $35 \mu \mathrm{g}$ of ethinyl estradiol. Contraception. 2006;73(3):223-228.

14. Jick SS, Hagberg KW, Hernandez RK, Kaye JA. Postmarketing study of Ortho Evra and levonorgestrel oral contraceptives containing hormonal contraceptives with $30 \mathrm{mcg}$ of ethinyl estradiol in relation to nonfatal venous thromboembolism. Contraception. 2010;81(1):16-21.

15. Cole JA, Norman H, Doherty M, Walker AM. Venous thromboembolism, myocardial infarction, and stroke among transdermal contraceptive system users. Obstet Gynecol. 2007;109(2 pt 1):339-346.

16. Dore DD, Norman H, Loughlin J, Seeger JD. Extended case-control study results on thromboembolic outcomes among transdermal contraceptive users. Contraception. 2010;81(5):408-413.

17. Daniels K, Daugherty J, Jones J, Mosher W. Current contraceptive use and variation by selected characteristics among women aged 15-44: United States, 2011-2013. Natl Health Stat Report. 2015;86:1-14.

18. Raine TR, Epstein LB, Harper CC, Brown BA, Boyer CB. Attitudes toward the vaginal ring and transdermal patch among adolescents and young women. $J$ Adolesc Health. 2009;45(3):262-267.

19. Sibai BM, Odlind V, Meador ML, Shangold GA, Fisher AC, Creasy GW. A comparative and pooled analysis of the safety and tolerability of the contraceptive patch (Ortho Evra/Evra). Fertil Steril. 2002;77(2 suppl 2): S19-S26.

20. Zacur HA, Hedon B, Mansour D, Shangold GA, Fisher AC, Creasy GW. Integrated summary of Ortho Evra/Evra contraceptive patch adhesion in varied climates and conditions. Fertil Steril. 2002;77(2 suppl 2): S32-S35.

21. Peipert JF, Zhao Q, Allsworth JE, et al. Continuation and satisfaction of reversible contraception. Obstet Gynecol. 2011;119(5): $1105-1113$

22. Harel Z, Riggs S, Vaz R, et al. Adolescents' experience with the combined estrogen and progestin transdermal contraceptive method Ortho Evra. J Pediatr Adolesc Gynecol. 2005;18(2):85-90.

23. Sucato GS, Land SR, Murray PJ, et al. Adolescents' experiences using the contraceptive patch versus pills. J Pediatr Adolesc Gynecol. 2011;24(4):197-203.

24. Raine TR, Foster-Rosales A, Upadhyay UD, et al. One-year contraceptive continuation and pregnancy in adolescent girls and women initiating hormonal contraceptives. Obstet Gynecol. 2011;117(2 pt 1): 363-371.
25. Maslyanskaya S, Coupey SM, Chhabra R, Khan UI. Predictors of early discontinuation of effective contraception by teens at high risk of pregnancy. J Pediatr Adolesc Gynecol. 2016;29(3):269-275.

26. Electronic Medicines Compendium; DataPharm Ltd. JanssenCilag Ltd. Evra transdermal patch: Pharmacodynamic properties. Available from: https://www.medicines.org.uk/emc/medicine/ 12124\#PHARMACODYNAMIC_PROPS. Accessed April 3, 2017.

27. Crosignani PG, Nappi C, Ronsini S, et al. Satisfaction and compliance in hormonal contraception: the result of a multicenter clinical study on women's experience with the ethinylestradiol/norelgestromin contraceptive patch in Italy. BMC Womens Health. 2009;9:18.

28. Jakimiuk AJ, Crosignani PG, Chernev T, et al. High levels of women's satisfaction and compliance with transdermal contraception: results from a European multinational, 6-month study. Gynecol Endocrinol. 2011;27(10):849-856.

29. Øjvind L, Nielsen LH, Skovlund CW, Løkkegaard E. Venous thrombosis in users of non-oral hormonal contraception: follow-up study, Denmark 2001-2010. BMJ. 2012;344:e2990.

30. Waellnitz K, Duijkers I, Klipping C, et al. A two-centre, open label, randomised study of ovulation inhibition with three transdermal contraceptive patches, each containing different amounts of ethinyl estradiol and gestodene in healthy, young women. J Obstet Gynaecol. 2016; 36(1):106-113.

31. Hofmann B, Reinecke I, Schuett B, Merz M, Zurth C. Pharmacokinetic overview of ethinyl estradiol dose and bioavailability using two transdermal contraceptive systems and a standard combined oral contraceptive. Int J Clin Pharmacol Ther. 2014;52(12):1059-1070.

32. Wiegratz I, Bassol S, Weisberg E, Mellinger U, Merz M. Effect of a low-dose contraceptive patch on efficacy, bleeding pattern, and safety: a 1-year, multicenter, open-label uncontrolled study. Reprod Sci. 2014; 21(12):1518-1525.

33. Gruber D, Skřivánek A, Serrani M, Lanius V, Merz M. A comparison of bleeding patterns and cycle control using two transdermal contraceptive systems: a multicenter, open-label, randomized study. Contraception. 2015;91(2):105-112.

34. Merz M, Kroll R, Lynen R, Bangerter K. Bleeding pattern and cycle control of a low-dose transdermal contraceptive patch compared with a combined oral contraceptive: a randomized study. Contraception. 2015; 91(2):113-120.

35. Kaunitz AM, Portman D, Westhoff CL, et al. Low-dose levonorgestrel and ethinyl estradiol patch and pill: a randomized control trial. Obstet Gynecol. 2014;123(2 pt 1):295-303.

36. Kaunitz AM, Portman D, Westhoff CL, Archer DF, Mishell DR Jr, Foegh M. Self-reported and verified compliance in a phase 3 clinical trial of a novel low-dose contraceptive patch and pill. Contraception. 2015;91(3):204-210.

37. Kaunitz AM, Archer DF, Mishell DR Jr, Foegh M. Safety and tolerability of a new low-dose contraceptive patch in obese and nonobese women. Am J Obstet Gynecol. 2015;212(3):318.e1-318.e8.

38. Archer DF, Stanczyk FZ, Rubin A, Foegh M. Pharmacokinetics and adhesion of the Agile transdermal contraceptive patch (AG200-15) during daily exposure to external conditions of heat, humidity and exercise. Contraception. 2013;87(2):212-219.

39. Kaunitz AM, Portman D, Westhoff CL, Mishell DR Jr, Archer DF, Foegh M. New contraceptive patch wearability assessed by investigators and participants in a randomized phase 3 study. Contraception. 2015;91(3):211-216.

40. Trussell J. Contraceptive failure in the United States. Contraception. 2011;83(5):397-404. 
International Journal of Women's Health

Dovepress

\section{Publish your work in this journal}

The International Journal of Women's Health is an international, peerreviewed open-access journal publishing original research, reports, editorials, reviews and commentaries on all aspects of women's healthcare including gynecology, obstetrics, and breast cancer. The manuscript management system is completely online and includes

Submit your manuscript here: http://www.dovepress.com/international-journal-of-womens-health-journa a very quick and fair peer-review system, which is all easy to use. Visit http://www.dovepress.com/testimonials.php to read real quotes from published authors. 